\title{
STIMULATION OF THE MOUSE HEPATIC MICROSOMAL ANILINE $p$-HYDROXYLASES BY ACETONE AND POLYAMINES
}

\author{
Arun P. Kulkarni and Ileana Gonzales \\ Toxicology Research Laboratory, Department of Environmental and Industrial Health, School of Public \\ Health, The University of Michigan, Ann Arbor, MI 48109-2029, U.S.A.
}

(Received 18 July 1984)

\begin{abstract}
The effects of acetone \pm spermine on the high (AH-I) and low (AH-II) affinity forms of aniline hydroxylases in the mouse hepatic microsomes were investigated under in vitro conditions.

2. The addition of either acetone or spermine alone stimulated both AH-I and AH-II activities at low concentration while some decline in stimulation was noted at higher concentrations.

3 . In the presence of both the modifiers the observed monoxygenation rates were greater than those produced by any one enhancer alone for AH-I and more than additive for AH-II.

4. The results suggest that the enhancement of aniline $p$-hydroxylation by the acetone and spermine in the mouse hepatic microsomes involves at least two separate and possibly interdependent mechanisms.
\end{abstract}

\section{INTRODUCTION}

The polyamines, spermine and spermidine are aliphatic nonprotein nitrogeneous bases of ubiquitous occurrence. In mammalian liver, polyamines occur in millimolar concentrations and exhibit dramatic fluctuations under a variety of experimental conditions (Tabor and Tabor, 1976).

Although the physiological role of polyamines is not at present clearly understood, available information suggests their influence on many cellular processes, especially those involving cell membranes (Tabor and Tabor, 1976). Earlier studies indicated that polyamines, in general, enhance cytochrome $P$-450-dependent hepatic microsomal mixed function oxidase system (Chapman, 1976; Kitada et al., 1980; Andersson et al., 1981; Dalet et al., 1983). In this regard, up to $40 \%$ increase in aniline $p$-hydroxylase activity of rat liver microsomes was observed in the presence of polyamines by Chapman (1976) and Kitada et al. (1980). Besides polyamines, a number of other chemicals have been shown to modify in vitro microsomal oxidation of xenobiotics (Cinti, 1978) of which acetone-enhancement of aniline $p$-hydroxylase has been repeatedly documented (Anders, 1968, 1969, 1972; Cinti, 1978; Anders and Gander, 1979).

The presence of (at least) two kinetically distinguishable forms of aniline $p$-hydroxylase, one with high affinity (low $\mathrm{Km}, \mathrm{AH}-\mathrm{I}$ ) and the other with low affinity (high $\mathrm{Km}, \mathrm{AH}-\mathrm{II}$ ) toward aniline have been described for hamster (McCoy, 1980) rat (Wada et al., 1968; Ebel, 1981; Bidlack and Lowery, 1982; Ortiz et al., 1982) and mouse (Wada et al., 1968) liver microsomes.

Earlier, Anders $(1969,1972)$ reported that both acetone and 2,2'-bipyridine share the same mechanism while ethylisocyanide brings about enhancement of aniline hydroxylation by another mechanism. Although a possible involvement of multiple forms of aniline $p$-hydroxylases was postulated in different studies (Wada et al., 1968; McCoy, 1980; Ebel, 1981; Bidlack and Lowery, 1982; Ortiz et al., 1982) the question whether AH-I or AH-II or both are affected by the addition of acetone was not examined. Since hepatic endoplasmic reticulum is in constant contact with polyamines it is of interest to examine acetoneenhancement phenomenon in the presence of polyamines. Our data suggest that separate but possibly inter-dependent mechanisms are involved in the stimulatory response of aniline $p$-hydroxylases to polyamines and acetone.

\section{Animals}

\section{MATERIALS AND METHODS}

Young male mice $\left(\mathrm{CD}_{1}\right.$ strain) of approximately $30 \mathrm{~g}$ body weight purchased from Charles River Laboratories were used. Animals were kept on a standard laboratory diet and tapwater ad libitum for at least 8 days before sacrifice.

\section{Tissue preparation}

The animals were stunned by a blow on the head, decapitated, exsanguinated and quickly hepatectomized. Liver microsomes were prepared by differential centrifugation from $20 \%$ homogenate in $50 \mathrm{mM}$ Tris buffer, $\mathrm{pH} 7.4$ containing $0.25 \mathrm{M}$ sucrose and $0.1 \mathrm{mM}$ EDTA as described previously (Kulkarni et al., 1975).

\section{Aniline $\mathrm{p}$-hydroxylase assay}

The standard assay mixtures containined $(3.0 \mathrm{ml}$ final volume) $1.0 \mathrm{mM} \mathrm{NADP}^{+}, 2.5 \mathrm{mM}$ glucose-6-phosphate; 1.5 units of glucose-6-phosphate dehydrogenase, $0.1 \mathrm{M}$ Tris buffer, $\mathrm{pH} 8.0 ; 2-3 \mathrm{mg}$ microsomal protein and indicated final concentrations of aniline, acetone and polyamine. Complete assay mixtures minus aniline were preincubated for $5 \mathrm{~min}$ at $37^{\circ} \mathrm{C}$. Reactions were initiated by the addition of substrate. Following incubation for $20 \mathrm{~min}$ at $37^{\circ} \mathrm{C}$, the amount of $p$-aminophenol produced was estimated as reported earlier (Kulkarni and Hodgson, 1982). Incubation mixtures without NADPH generating system served as controls. Protein was estimated by the method of Lowry et al. (1951) using crystalline bovine serum albumin, fraction $\mathrm{V}$, as standard. The results are reported as relative activity (Mean \pm SEM). Relative Activity $=$

Specific Activity in the Presence of Acetone \pm Polyamine Basal Activity 
where basal activity represents the specific activity (nmol of $p$-aminophenol produced $/ \mathrm{hr} / \mathrm{mg}$ microsomal protein) observed in the absence of both acetone and polyamine.

\section{RESULTS AND DISCUSSION}

Wada et al. (1968) observed a biphasic response curve when reciprocal of the velocity was plotted against the reciprocal of aniline concentration and concluded the existance of two or more forms of aniline $p$-hydroxylase activity in the mouse liver microsomes. Recently, McCoy (1980) also reported similar observations and proposed the presence of two aniline $p$-hydroxylases in the hamster liver microsomes. He recommended the use of 0.1 and $20 \mathrm{mM}$ aniline in assay media to estimate the high affinity form (AH-I) and total aniline $p$-hydroxylase (TAH) activity, respectively. The calculated difference between the two activities was presumed to represent the titer of the low affinity form (AH-II). Consistent with this view, Ortiz et al. (1982) employed 0.1 and $16 \mathrm{mM}$ aniline to evaluate $\mathrm{AH}-\mathrm{I}, \mathrm{TAH}$ and AH-II activities in the rat liver microsomes. Other induction and inhibition studies (McCoy, 1980; Ebel, 1981; Bidlack and Lowery, 1982) also support the concept of multiplicity of aniline $p$-hydroxylase in the mammalian hepatic microsomes.

Previous reports have shown the relative titer of AH-II in hepatic microsomes to be about $40 \%$ in rats (Ortiz et al., 1982) and 40-64\% in hamster (McCoy, 1980). In our study we used 0.1 and $15 \mathrm{mM}$ aniline and found that of the total mouse hepatic microsomal $p$-hydroxylase activity $(29.1 \pm 2.0 \mathrm{nmol}$ of $p$ aminophenol formed $/ \mathrm{hr} / \mathrm{mg}$ microsomal protein), the mean relative contribution of $\mathrm{AH}-\mathrm{II}$ was about $43 \%$ and it varied between 23 and $51 \%$ in different microsomal preparations $(n=15$ each). In view of the considerable variation in $\mathrm{AH}-\mathrm{II}$ activity, no attempt was made to establish $K_{m}$ and $V_{\max }$ values for the isozymes of aniline hydroxylase present in the mouse hepatic microsomes. It should also be pointed out that the concept of multiplicity of aniline $p$ hydroxylase is not yet firmly established by, for example, reconstitution studies utilizing different forms of purified cytochrome $P-450$ species specific for aniline $p$-hydroxylation to evaluate relative affinity and oxidation rates or by appropriate antibody experiments. Therefore, the usage of the terms $\mathrm{AH}-\mathrm{I}$ and $\mathrm{AH}-\mathrm{II}$ in this paper although is in accordance with current convention, possibility exists that these two forms actually represent one or more species of cytochrome $P-450$.

Consistant with previous reports (Anders, 1968, 1969, 1972; Anders and Gander, 1979; Vianio and Hanninen, 1972; Powis et al., 1977; Bidlack and Lowery, 1982) acetone alone was found to stimulate TAH (Fig. 1). Based on observations that acetone increases both $K_{m}$ for aniline and $V_{\max }$ in rat liver microsomes Anders (1968) suggested a possibility of unique susceptibility of $\mathrm{AH}-\mathrm{I}$ to acetone inhibition leading to its suppression concomitant with an increased expression of AH-II activity. However, our data (Figs 2 and 3 ) on the mouse hepatic microsomes indicate that both the isozymes are stimulated by acetone. The magnitude of AH-I and AH-II stimulation however, was not equal and was related to acetone concentration used. A maximum increase of

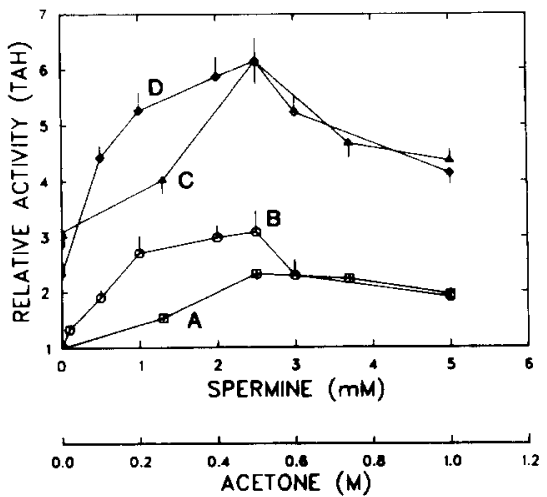

Fig. 1. Effects of acetone and spermine on the total aniline $p$-hydroxylase activity (TAH) of the mouse hepatic microsomes. The incubation mixtures contained microsomes, $15 \mathrm{mM}$ aniline, NADPH generating system and either $0-0.9 \mathrm{M}$ acetone (line $\mathrm{A}$ ); $0-5.0 \mathrm{mM}$ spermine (line $\mathrm{B}$ ); $2.5 \mathrm{mM}$ spermine and $0-0.9 \mathrm{M}$ spermine (line $\mathrm{C}$ ) or $0.45 \mathrm{M}$ acetone and 0-5.0 mM spermine (line D). Each point represents mean of relative specific activity $(=1$ in control $) \pm S E$ ( $N=$ 4-8 separate experiments). The basal (TAH) activity in the absence of both acetone and spermine was $29.1 \pm 2.0 \mathrm{nmol}$ of $p$-aminophenol produced $/ \mathrm{hr} / \mathrm{mg}$ microsomal protein $(N=15)$. See Materials and Methods for further details.

about $160 \%$ in AH-I activity (Fig. 2, line A) and of about $100 \%$ in AH-II activity (Fig. 3, line A) over control values was observed in the presence of $0.45 \mathrm{M}$ and $0.68 \mathrm{M}$ acetone respectively. Apparently, these observations do not represent a species-specific difference in response to acetone since with rat liver microsomes also, Bidlack and Lowery (1982) observed acetone to produce a general increase in hydroxylase activity at a lower aniline concentration and a specific increase in activity with $>0.75 \mathrm{mM}$

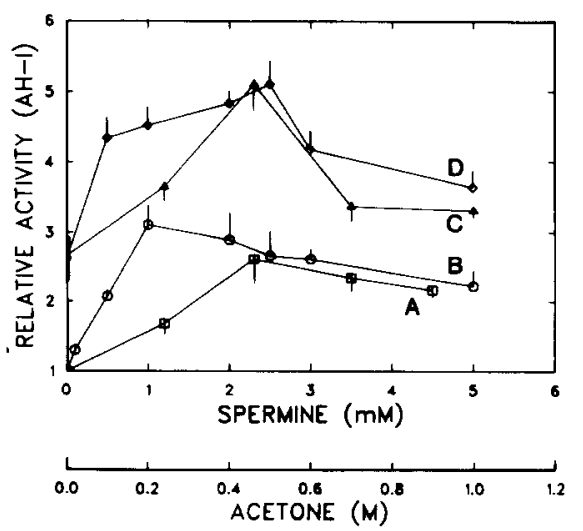

Fig. 2. Effects of acetone and spermine on the AH-I activity of the mouse hepatic microsomes. The assay media contained microsomes, $0.1 \mathrm{mM}$ aniline, NADPH generating system and either $0-0.9 \mathrm{M}$ acetone (line A); $0-5.0 \mathrm{mM}$ spermine (line $\mathrm{B}$ ); $2.5 \mathrm{mM}$ spermine and $0-0.9 \mathrm{M}$ acetone (line $\mathrm{C}$ ) or $0.45 \mathrm{M}$ acetone and $0-5.0 \mathrm{mM}$ spermine (line $\mathrm{D}$ ). each point represents mean of relative specific activity $(=1.0$ control) \pm SE $(N=4-8$ separate experiments). The basal AH-I activity in the absence of both acetone and spermine was $16.5 \pm 1.3 \mathrm{nmol}$ of $p$-aminophenol produced $/ \mathrm{hr} / \mathrm{mg} \mathrm{mi-}$ crosomal protein $N=15$ ). See Materials and Methods for futher details. 
aniline. Similarly, Anders and Gander (1979) reported a marked dependence of acetone enhancement on aniline concentration and the stimulation, in general, was greater in NADPH/oxygen as well as cumene hydroperoxide supported reactions when lower aniline concentrations were used.

Although in all the previous investigations (Anders, 1968, 1969, 1972; Anders and Gander, 1979; Vianio and Hanninen, 1972; Powis et al., 1977; Bidlack and Lowery, 1982), only the stimulatory effect of acetone had been the focal point of proposed mechanisms, our data (Figs 1-3) indicate that at higher acetone concentrations, the activity-response curves for TAH, AH-I as well as AH-II do not plateau but rather exhibit a gradual decline in stimulation. This was also noted by Anders (1968) with rat liver microsomes and by Kitada et al. (1983) in the reconstituted systems. Our data, considered together with the known inhibitory effects of acetone on several other monooxygenase reactions (Cinti, 1978; Kawalek and Andrews, 1980) suggest that at any given concentration acetone exerts both stimulatory as well as inhibitory effects on aniline $p$ hydroxylation. The observed activity, therefore, represents the net balance between these two opposing effects. In the mouse liver microsomes, the equilibrium appears to be in favor of maximum stimulation when an acetone concentration of about $0.45 \mathrm{M}$ in the case of AH-I and $0.68 \mathrm{M}$ in the case of AH-II was used.

It is currently believed that acetone enhancement of aniline $p$-hydroxylation probably involves either facilitated introduction of second electron (Powis et al., 1977), a site for formation or insertion of activated oxygen into the substrate (Anders and Gander, 1979) and/or multiple forms of aniline $p$-hydroxylase

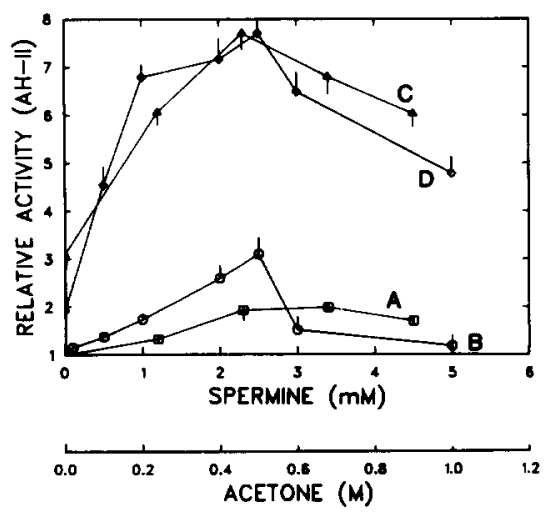

Fig. 3. Effects of acetone and spermine on the AH-II activity of the mouse hepatic microsomes. The AH-II activity was calculated by substracting AH-I activity from TAH activity observed in the assay media containing microsomes, 0.1 or $15 \mathrm{mM}$ aniline, NADPH generating system and either $0-0.9 \mathrm{M}$ acetone (line A); $0-5.0 \mathrm{mM}$ spermine (line B); $2.5 \mathrm{mM}$ spermine and $0-0.9 \mathrm{M}$ acetone (line $\mathrm{C}$ ) or $0.45 \mathrm{M}$ acetone and $0-5.0 \mathrm{mM}$ spermine. Each value represents mean of relative AH-II activity ( $=1.0$ in control \pm SEM). The calculated basal AH-II activity in the absence of both acetone and spermine was $12.5 \pm 2.8 \mathrm{nmol}$ of $p$ aminophenol produced $/ \mathrm{hr} / \mathrm{mg}$ microsomal protein $(N=15)$. The data on TAH and AH-I activity are given in Figs 1 and 2. See text for further details.
(Bidlack and Lowery, 1982). For a variety of reasons, it is believed that the type-I and type-II binding of aniline to cytochrome $P-450$, and lipid peroxidation do not play a significant role (Cinti, 1978; Anders and Gander, 1979; Bidlack and Lowery, 1982). Conflicting results have been reported in the literature as regards the role of cytochrome $P-450$ reduction rate in the acetone stimulated aniline hydroxylation. Thus Vainio and Hanninen (1972) reported that acetone partly reverses the inhibition of NADPH-cytochrome $P-450$ reductase produced by aniline and suggested that this might partly explain the enhancing effects of acetone. The results of reconstitution studies (Kitada et al., 1983) with phenobarbital inducible form of cytochrome $P-450$ are in favor of this view. In contrast to this, Powis et al. (1977) reported that acetone inhibits NADPHcytochrome $P-450$ reductase and the inhibitory effects were not additive in the presence of aniline. A possibility that this reductase may not be the locus of enhancement is apparent from the reported stimulation of hepatic microsomal aniline hydroxylation by acetone in the cumene hydroperoxide-mediated reaction (Anders and Gander, 1979). However, it should be pointed out that Kitada et al. (1983) failed to observe such a stimulation in the cumene hydroperoxide-supplemented reconstituted systems containing either cytochrome $P-450$ or $P-448$.

Similar to acetone, spermine alone also caused significant enhancement of aniline $p$-hydroxylase activity of the mouse hepatic microsomes (Figs 1-3). The spermine concentration as low as $100 \mu \mathrm{M}$ produced a detectable increase $(15-30 \%)$ in activity. In contrast to acetone which caused relatively more stimulation of AH-I activity, the stimulatory effects of spermine were nearly equal on both the forms (Figs 2 and 3). A maximum of about a 3-fold increase was observed when optimal concentration of spermine was present in the incubation medium. Earlier, Chapman (1976) and Kitada et al. (1983) reported, respectively, only about a 40 and $8 \%$ increase in aniline $p$-hydroxylation by rat liver preparations in the presence of 1-5 mM spermine. A higher degree of stimulation noted in our study with the mouse hepatic microsomes, therefore, may be due to differences in the methodology or represents a true species-specific difference.

The effects of both acetone and spermine on aniline $p$-hydroxylation are also shown in Figs 1-3. In each case, the observed value for TAH activity (Fig. 1) was found to be either equal to or greater than the expected value (based on activity noted in the presence of either acetone or spermine). The two forms of the enzyme, however, were not equally affected and as compared to AH-I the magnitude of AH-II stimulation was much greater (Figs 2 and 3 lines $\mathrm{C}$ and D). Except for the reaction media containing $0.45 \mathrm{M}$ acetone, the results, in general show a simple additive or less than additive effect on AH-I (Fig. 2) and greater than additive effects on AH-II (Fig. 3). The decrease in the magnitude of stimulation of TAH, $\mathrm{AH}-\mathrm{I}$ and $\mathrm{AH}-\mathrm{II}$ when $>2.5 \mathrm{mM}$ spermine was used in the presence or absence of acetone (Figs 1-3; lines $B$ and D) may be related to aggregating effects of spermine on microsomal vesicles (Tadolini, 1980). Such an alteration may decrease the total number of 
accessible binding sites which in turn will render availability of NADPH and possibly aniline rate limiting in overall monooxygenation process.

When aniline concentration was varied $(0.1-15.0 \mathrm{mM})$ in the reaction media containing $2.5 \mathrm{mM}$ spermine and $0.45 \mathrm{M}$ acetone, a maximum stimulation of about 6-fold was observed with $2.5 \mathrm{mM}$ aniline (Fig. 4, line A). The magnitude of stimulation did not increase further with higher aniline concentration. This suggests a general increased affinity of isozymes for aniline. Under these conditions, $2.5 \mathrm{mM}$ aniline apparently saturates both the isozymes, and the observed rate, therefore, represents a total aniline $p$-hydroxylase activity. The results also suggest that in the presence of both acetone and spermine, high aniline concentration (5.0-15.0 mM) is not inhibitory. In the absence of enhancing agents, aniline has been shown to cause substrate inhibition at high concentrations (Ebel, 1981). The data given in Fig. 4, indicate that at $2.5 \mathrm{mM}$ aniline, maxımum stimulation is observed when $0.45 \mathrm{M}$ acetone (line $\mathrm{C}$ ) or $2.5 \mathrm{mM}$ spermine (line B) alone was used. However, in the presence of both the enhancing agents greater than additive effects were observed. The stimulation was dependent upon amount of acetone present into the incubation media and peaked at $0.45 \mathrm{M}$ (line D).

Similar experiments were conducted in the presence of spermidine and putrescine (the data not shown). A maximum stimulation of about 5 fold in the aniline $p$-hydroxylases was observed in the presence of $0.68 \mathrm{M}$ acetone, $3.0 \mathrm{mM}$ spermidine and $2.5 \mathrm{mM}$ aniline. A similarity between the effects of spermidine and spermine includes that both caused

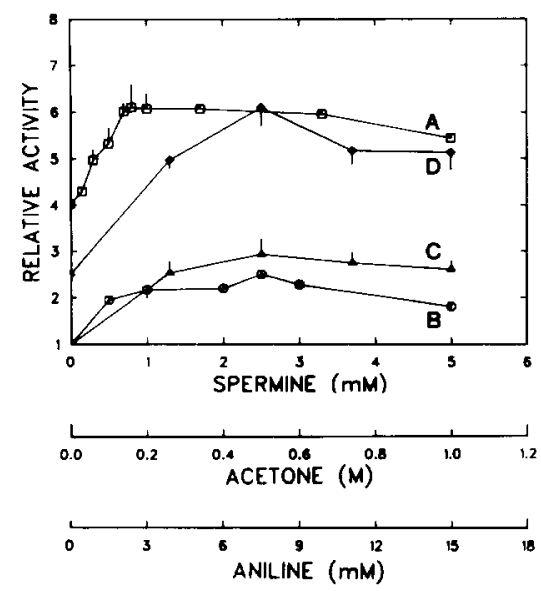

Fig. 4. Effects of acetone and spermine on the aniline $p$-hydroxylase activity of the mouse hepatic microsomes. The incubation media contained microsomes, NADPH generating system and either $2.5 \mathrm{mM}$ spermine, $0.45 \mathrm{M}$ acetone and $0.1-15 \mathrm{mM}$ aniline (line $\mathrm{A}$ ); $2.5 \mathrm{mM}$ aniline and $0-5 \mathrm{mM}$ spermine (line B); $2.5 \mathrm{mM}$ aniline and $0-0.9 \mathrm{M}$ acetone (line-C); or $2.5 \mathrm{mM}$ aniline, $2.5 \mathrm{mM}$ spermine and $0-0.9 \mathrm{M}$ acetone (line D). Each point represents mean of relative specific activity $(=1.0$ in control $) \pm S E$. The basal activities in the absence of both acetone and spermine using 2.5 (lines B, C and D) and 0.1 (line A) $\mathrm{mM}$ aniline were $36.6 \pm 4.1$ and $21.2 \pm 2.7 \mathrm{nmol}$ of $p$-aminophenol produced $/ \mathrm{hr} / \mathrm{mg}$ microsomal protein $(N=4-8$ separate experiments). See text for further details. relatively more stimulation of AH-II activity. Only marginal increase in the aniline $p$-hydroxylases were observed in the presence of low concentrations of putrescine while high levels were found to be inhibitory.

Kitada et al. (1980) observed no significant increase in ethylmorphine $\mathrm{N}$-demethylation by either spermine, spermidine or putrescine in a reconstituted monooxygenase system consisting of purified cytochrome $P-450$, NADPH-cytochrome $P-450$ reductase, both isolated from phenobarbital pretreated rats and either extracted lipids or dilauroylL-3-phosphatidylcholine. However, stimulation was observed when hepatic microsomes were used. In contrast to these results, Andersson et al. (1981) working with a reconstituted system containing cytochrome $P-450 \mathrm{LM}_{2}$ or $\mathrm{LM}_{4}$, NADPH-cytochrome $P-450$ reductase, both isolated from either untreated or phenobarbital pretreated rabbits and dilauroylglyceryl-3-phosphorylcholine observed 3- to 4-fold stimulation of benzphetamine and 7-ethoxycoumarin dealkylation by spermine. Similar data were also reported for the monooxygenation of benzphetamine, 7-ethoxycoumarin, benzo(a)pyrene and testosterone by liver microsomes from human and untreated or phenobarbital pretreated rabbits (Dalet et al., 1983). These results suggest some specificity in the stimulation of monooxygenation reactions by polyamines. In mammalian hepatic microsomes phosphatidyl serine is localized primarily on the cytosolic side (Nilsson and Dallner, 1975) giving this surface a higher negative charge density than the canalicular surface. Neutralization of these negative charges by organic polycations such as polyamines is expected to bring about stabilizing effects on membranes. Thus, removal of physical constraints from microsomal membranes and/or an allosteric effect of polyamines on the cytochrome $P-450$ molecule may be involved. Apparently, the specificity and the magnitude of this effect depend not only upon the species of cytochrome $P-450$ in question but also upon the substrate and other factors such as its surrounding phospholipid environment.

The actual underlying molecular events leading to increased enzyme activity by polyamines are unclear at present and do not probably include effects on substrate binding to cytochrome $P-450$ (Kitada et al., 1980; Andersson et al., 1981; Dalet et al., 1983; Osimitz and Kulkarni, 1984). Recently Kitada et al. (1983) have proposed that the inhibitory effects of polyamines on lipid peroxidation might be responsible for their stimulatory effects on monooxygenation reactions. The role of this factor in the observed stimulation of aniline $p$-hydroxylation is questionable since we (Kulkarni and Hodgson, 1981) have earlier shown that in the mouse hepatic microsomes aniline itself inhibits lipid peroxidation. Our laboratory has investigated the effects of polyamines on the different steps of cytochrome $P-450$-mediated reaction in the mouse hepatic microsomes (Osimitz and Kulkarni, 1984). For example, spermidine significantly increased the rate of NADPH oxidation and NADPH-dependent cytochrome $P-450$ reduction while redox behaviour of cytochrome $b_{5}$ and $\mathrm{H}_{2} \mathrm{O}_{2}$ generation rates were unaffected suggesting that efficient coupling of NADPH utilization may be 
one of the mechanisms of polyamine-caused stimulation of aniline hydroxylation. In view of the complexity noted in the interpretation of data from preliminary experiments, further attempts to elucidate the effects of acetone-polyamine combination on the individual steps of monooxygenation reaction cycle were discontinued.

The observed additive effects clearly suggest that two separate mechanisms are involved in the enhancement of aniline $p$-hydroxylation by acetone and polyamines. The greater than additive effects indicate inter-dependency of the polyamine stimulation on acetone effects. Anders and Gander (1979) suggested that acetone removes a rate limiting factor by exerting its effect at or near the formation or insertion of the activation oxygen. The reported lack of stimulation by polyamines of either $\mathrm{H}_{2} \mathrm{O}_{2}$ (Kitada et al., 1980) or cumene hydroperoxide (Dalet et al., 1983) supported monooxygenation reactions and the fact that some of the experiments described in this paper were performed in the presence of acetone concentrations that produced maximum stimulation (in the absence of polyamines), suggest that the polyaminecaused further stimulation of aniline $p$-hydroxylation involves additional mechanism(s) other than the sites for oxygen activation or insertion. Alternately, it must be presumed that these steps remain rate limiting in NADPH supported systems even in the presence of acetone.

Dalet et al. (1983) noted increased rates of 7-ethoxycoumarin $\mathrm{O}$-deethylation by rabbit liver microsomes due to relatively smaller spermine addition to reaction media containing NADH as well as NADPH. Based on the observations that the NADH-NADPH synergism can only be observed in the presence of acetone Powis et al. (1977) postulated that acetone facilitates the transfer of second electron to oxyferrous complex and this may be the rate limiting step in aniline $p$-hydroxylation. These authors also suggested that enhancing agents relieve the aniline caused inhibition of transfer of second electron derived from NADPH. Earlier Oshino and Sato (1971) reported enhancement of microsomal cytochrome $b_{5}$ reoxidation by aniline as well as $p$-aminophenol, the oxidation product of aniline. An increase in aniline hydroxylation rate is expected if the enhancing agents produce "sparing effects" on electrons derived from NADPH by blocking uncoupling caused by aniline and $p$-aminophenol. Although the reported stimulation of NADH oxidation by the enhancing agents (Powis et al., 1977) is in favor of this hypothesis, Stevens et al. (1972) did not observe inhibition of aniline $p$-hydroxylation by the exogenously added excess $p$-aminophenol. In any case the role of cytochrome $b_{5}$ becomes obscure since polyamines do not alter reduction and reoxidation rate of cytochrome $b_{5}$ in the mouse hepatic microsomes (Osimitz and Kulkarni, 1984) but can stimulate monooxygenation reaction in the reconstituted system in the absence of this hemoprotein (Andersson et al., 1981; Dalet et al., 1983). However, a faster introduction of second electron via NADPHcytochrome $P-450$ reductase is likely since we (Osimitz and Kulkarni, 1984) have observed that polyamines significantly stimulate this activity in the mouse hepatic microsomes. Thus, in conclusion it is believed that the stimulation of aniline $p$ hydroxylation in the presence of both acetone and polyamines involves more than one of these mechanisms which operate in a concerted fashion. The results also clearly indicate that mammalian hepatic microsomes possess much greater capacity to hydroxylate aniline than previously realized by routine assays in the presence of either NADPH alone or NADPH plus acetone.

Acknowledgement-This work was supported in part by Grant T32 ES07062 from the U.S. Public Health Service, National Institute of Health.

\section{REFERENCES}

Anders M. W. (1968) Acetone enhancement of microsomal aniline para-hydroxylase activity. Archs Biochem. Biophys. 126, 269-275.

Anders M. W. (1969) Stimulation in vitro of microsomal aniline hydroxylation by $2,2^{\prime}$-bipyridine. Biochem. Pharmac. 18, 2561-2565.

Anders M. W. (1972) Effect of phenobarbital and 3-methylcholanthrene administration of the in vitro enhancement of microsomal aromatic hydroxylation. Archs Biochem. Biophys. 153, 502-507.

Anders M. W. and Gander J. E. (1979) Acetone enhancement of cumene hydroperoxide supported microsomal aniline hydroxylation. Life Sci. 25, 1085-1090.

Andersson K. K., Dalet C., Bonfils C. and Maurel P. (1981) Effect of polyamine on microsomal cytochrome $P-450$ : stimulation of rate and improved coupling of NADPH oxidation to hydroxylation. Biochem. biophys. Res. Commun. 98, 311-316.

Bidlack W. R. and Lowery G. L. (1982) Multiple drug metabolism: p-nitroanisole reversal of acetone enhanced aniline hydronylation. Biochem. Pharmac. 31, 311-317.

Chapman S. K. (1976) Polyamines and drug onidations. Drug Metab. Dispos. 4, 417-422.

Cinti D. L. (1978) Agents activating the liver microsomal mixed function oxidase system. Pharmac. Ther. 2, 727-749.

Dalet C., Andersson K. K., Beluche I. D., Bonfils C. and Maurel P. (1983) Polyamines as modulators of drug oxidation reactions catalyzed by cytochrome P-450 from liver microsomes. Biochem. Pharmac. 32, 593-601.

Ebel R. E. (1981) Hepatic microsomal aniline phydroxylase: Substrate inhibition by aniline. Drug Metab. Dispos. 9, 578-579.

Kawalek J. C. and Andrews A. W. (1980) The effect of solvents on drug metabolism in vitro. Drug Metab. Dispos. 8, 380-384.

Kitada M., Yamaguchi N., Igarashi K., Hirose S. and Kitagawa $H$. (1980) Effects of polyamines on ethylmorphine N-demethylation in rat liver microsomes. Jap. J. Pharmac. 30, 579-586.

Kitada M., Ando M., Ohmori S., Kabuto S., Kamataki T. and Kitagawa H. (1983) Effect of acetone on aniline hydroxylation by a reconstituted system. Biochem. Pharmac. 32, 3151-3155.

Kulkarni A. P., Mailman R. B. and Hodgson E. (1975) Cytochrome $P-450$ optical difference spectra of insecticides: A comparative study. J. Agric. Fd Chem. 23, 177-183.

Kulkarni A. P. and Hodgson E. (1981) A comparison of NADPH and cumene hydroperoxide-stimulated lipid peroxidation in mouse hepatic microsomes. Int. J. Biochem. 13, 811-816.

Kulkarni A. P. and Hodgson E. (1982) Mouse liver microsomal hexose-6-phosphate dehydrogenease: NADPH generation and utilization in monooxygenation reactions. Biochem. Pharmac, 31, 1131-1137. 
Lowry O. H., Rosebrough N. J., Farr A. L. and Randall R. J. (1951) Protein measurement with the Folin phenol reagent. J. biol. Chem. 193, 265-275.

McCoy G. D. (1980) Differential effects of ethanol and other inducers of drug metabolism on the two forms of hamster liver microsomal aniline hydroxylase. Biochem. Pharmac. 29, 685-688.

Nilsson O. and Dallner G. (1975) Distribution of constitutive enzymes and phospholipids in microsomal membranes of rat liver. FEBS Lett. 58, 190-193.

Ortiz E., Patel J. M. Leibman K. C. (1982) Specific inactivation of aniline hydroxylase by a reactive intermediate formed during acrylamide biotransformation by rat liver microsomes. Biological Reactive IntermedicatesChemical Mechanisms and Biological Effects Conference, The University of Surrey, Guildford, U.K. Adv. exp. Med. Biol. 136B, 1221-1227.

Oshino N. and Sato R. (1971) Stimulation by phenols of the reoxidation microsomal bound cytochrome $b_{5}$ and its implication to fatty acid disaturation. J. Biochem. Tokyo 69, $169-180$.

Osimitz T. G. and Kulkarni A. P. (1984) Polyamine effects on cytochrome $P-450$ and flavin containing monooxygenase mediated oxidation of xenobiotics. Drug Metab. Dispos. In press.

Powis G., Lyon L. and McKillop D. (1977) NADH synergism of microsomal aniline metabolism in the presence of enhancing agents. Biochem. Pharmac. 26, 137-141.

Stevens J. T., McPhillips J. J. and Stitzel R. E. (1972) In vitro enhancement of aniline metabolism by paraoxon. Toxic. appl. Pharmac. 23, 208-215.

Tabor C. W. and Tabor H. (1976) 1,4-Diaminobutane (putrescine), spermidine and spermine. A. Rev. Biochem. 45, 285-306.

Tadolini B. (1980) Polyamines effect on subcellular fractionation of rat liver homogenate. Biochem. biophys. Res. Commun. 92, 598-605.

Vianio H. and Hanninen O. (1972) Enhancement of aniline $p$-hydroxylation by acetone in rat liver microsomes. Xenobiotica 2, 259-267.

Wada F., Shimakawa H., Takasugi M., Kotake T. and Sakamoto Y. (1968) Effects of steroid hormones on drug-metabolizing enzyme systems in liver microsomes. $J$. Biochem. Tokyo 64, 109-113. 